\title{
Diagnosis-Based Differences in Response of Global Ventricular Performance to Modified Ultrafiltration in Children
}

\author{
Osami Honjo, MD; Satoru Osaki, MD; Yasuhiro Kotani, MD; \\ Teiji Akagi, MD*; Shunji Sano, MD
}

\begin{abstract}
Background: To determine diagnosis-based differences in the response of global ventricular performance to modified ultrafiltration (MUF) using transesophageal echocardiography during congenital heart surgery.
\end{abstract}

\begin{abstract}
Methods and Results: The study included 38 children with atrial septal defect $(n=10)$, ventricular septal defect (VSD) $(n=8)$, tetralogy of Fallot (TOF) $(n=9)$, or a single ventricle $(n=11)$. Arteriovenous MUF was performed for 10-15 min after cardiopulmonary bypass (CPB). The myocardial performance index (MPI) of the systemic ventricles and the \% change in MPI before and after MUF were assessed. Impairment of MPI was noted at termination of CPB compared with baseline values in the VSD and TOF groups $(\mathrm{P}<0.05)$. MUF resulted in an improvement in MPI in all groups $(P<0.01)$. There was a weak correlation between aortic cross-clamping or CPB time, and the degree of improvement in MPI $(r=-0.385, P=0.019 ; r=-0.348, P=0.037$, respectively). MUF improved fractional shortening in all groups $(\mathrm{P}<0.05)$ and reversed abnormal relaxation in the VSD and TOF groups.
\end{abstract}

Conclusions: Modified ultrafiltration ameliorated MPI in all groups, indicating improved systemic ventricular function with MUF. The MPI recovery rate differed among the groups. MUF may be particularly useful for restoring the global ventricular performance of patients undergoing longer CPB and may have minimal advantages for simple open-heart surgery. (Circ J 2010; 74: 86-92)

Key Words: Cardiopulmonary bypass; Congenital heart disease; Echocardiography; Modified ultrafiltration

A dvances in the technology of cardiopulmonary bypass (CPB) have improved the clinical outcomes of patients with congenital heart diseases undergoing open-heart surgery. Nonetheless, they are inevitably exposed to hypothermia and hemodilution, which cause systemic inflammation, accumulation of body water, and subsequent organ dysfunction. ${ }^{1}$ To minimize these adverse effects of $\mathrm{CPB}$, modified ultrafiltration (MUF) was introduced by Naik et $\mathrm{al},{ }^{2}$ and has become an essential perfusion strategy. ${ }^{3}$ Potential mechanisms underlying the clinical benefits of MUF include reduction of total body water accumulation, reversal of hemodilution, ${ }^{2,3}$ and modulation of the systemic inflammatory mediators. ${ }^{3,4}$ Recent clinical studies have shown that MUF improves pulmonary function, ${ }^{5}$ decreases postoperative bleeding ${ }^{6}$ and improves ventricular function. ${ }^{7,8}$

Improved ventricular function has been considered one of the most important clinical benefits of MUF. Davies et al showed that MUF resulted in an increase in the slope of the preload recruitable stroke work index of the left ventricle $(\mathrm{LV}){ }^{7}$ Furthermore, Chaturvedi et al reported improved systolic LV function in the MUF group using load-independent indices of LV function. ${ }^{8}$ Some controversies remain over whether MUF ameliorates diastolic dysfunction: 1 study showed no evidence of improvement in diastolic function in a conductance catheter study, ${ }^{8}$ but another showed a decrease in ventricular end-diastolic pressure and an increase in end-diastolic length, indicating improvement in ventricular compliance. ${ }^{7}$ These conflicting results might be partly related to the heterogeneity of the patient populations in those previous studies, because the degree of postoperative ventricular dysfunction, as well as the effects of MUF, on the damaged ventricles could be dependent on the length of myocardial ischemia and CPB and/or the magnitude of the surgical procedures required, as well as preoperative cardiac morphology and physiology.

The Doppler-derived myocardial performance index (MPI), which is also known as the Tei index, is a measure of the combined systolic and diastolic function of the ventricles. $^{9}$ This index is capable of assessing left or right ventricular global function because it is independent of geometric

Received April 15, 2009; revised manuscript received September 8, 2009; accepted September 10, 2009; released online November 27, 2009 Time for primary review: 27 days

Department of Cardiovascular Surgery, *Cardiac Care Unit, Okayama University Graduate School of Medicine, Dentistry and Pharmaceutical Sciences, Okayama, Japan

Mailing address: Osami Honjo, MD, Division of Cardiovascular Surgery, The Hospital for Sick Children, Toronto, 555 University Avenue, Toronto, ON, M5G, 1X8, Canada. E-mail: osami.honjo@ sickkids.ca

ISSN-1346-9843 doi:10.1253/circj.CJ-09-0248

All rights are reserved to the Japanese Circulation Society. For permissions, please e-mail: cj@j-circ.or.jp 


\begin{tabular}{|c|c|c|c|c|c|c|c|}
\hline Group & $\mathbf{n}$ & Age (years) & Body weight (kg) & AoX (min) & CPB (min) & MUF (min) & Transfusion (\%) \\
\hline ASD & 10 & $6.9 \pm 4.1$ & $24.2 \pm 14.1$ & $13 \pm 10$ & $35 \pm 15$ & $14 \pm 3$ & $0 / 10(0)$ \\
\hline VSD & 8 & $3.8 \pm 3.9$ & $18.2 \pm 15.2$ & $34 \pm 12$ & $58 \pm 15$ & $13 \pm 2$ & $1 / 8(13)$ \\
\hline TOF & 9 & $2.5 \pm 2.1$ & $11.2 \pm 3.2$ & $96 \pm 21$ & $128 \pm 44$ & $13 \pm 2$ & $1 / 9(11)$ \\
\hline SLV & 6 & $4.0 \pm 2.8$ & $14.7 \pm 3.9$ & $47 \pm 28$ & $92 \pm 23$ & $12 \pm 2$ & $0 / 5(0)$ \\
\hline SRV & 5 & $2.7 \pm 3.2$ & $11.3 \pm 5.8$ & $51 \pm 21$ & $89 \pm 18$ & $13 \pm 2$ & 2/6 (33) \\
\hline$P$ value & NS & $<0.01^{*}$ & $<0.05^{*}, 9, \S$ & $<0.001^{*, q, \S, t, \neq}$ & $<0.001^{*, q, \S, t, \ddagger}$ & NS & NS \\
\hline
\end{tabular}

*ASD vs TOF; †VSD vs TOF; `VSD vs SLV; §ASD vs SLV; १ASD vs SRV.

AoX, aortic cross-clamping time; CPB, cardiopulmonary bypass; MUF, modified ultrafiltration; ASD, atrial septal defect; VSD, ventricular septal defect; TOF, tetralogy of Fallot; SLV, single left ventricle; SRV, single right ventricle.

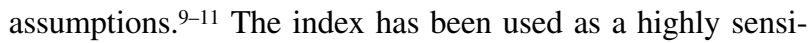
tive indicator of ventricular function in patients with various congenital heart defects measured as having uni- or biventricular physiology by transthoracic echocardiography. ${ }^{12,13}$ The MPI has also been measured by intraoperative transesophageal echocardiography (ITEE) to evaluate various intraoperative interventions in patients undergoing open-heart surgery. ${ }^{14,15}$ We hypothesized that an intraoperative real-time measurement of MPI might allow us to better demonstrate the effect of MUF on systemic ventricular function, so in this study, we divided the entire cohort into 5 subgroups: atrial septal defect (ASD), ventricular septal defect (VSD), tetralogy of Fallot (TOF), single left ventricle (SLV) and single right ventricle (SRV), with the aim of determining by means of the Doppler-derived MPI the diagnosis-based differences in the response of systemic ventricular performance to MUF in children undergoing open-heart surgery.

\section{Methods}

\section{Patient Population}

We conducted a study of children who underwent open-heart surgery from January 2003 to July 2005 at Okayama University Hospital. Data were collected in a prospective manner as part of routine ITEE, and were analyzed retrospectively after the study period. The Institutional Review Board approved the study and patient consent was waived. The 38 children who were enrolled included patients with $\operatorname{ASD}(n=10), \operatorname{VSD}(n=8), \operatorname{TOF}(n=9), \operatorname{SLV}(n=6$; tricuspid atresia, $n=3$; transposition of great arteries with large VSD, $\mathrm{n}=2$; pulmonary atresia with intact ventricular septum, $\mathrm{n}=1$ ), and SRV ( $n=5$; hypoplastic left heart syndrome, $n=3$; double outlet right ventricle with hypoplastic left ventricle, $n=2$ ). Age and body weight of each group are shown in Table 1. All patients underwent primary total repair, except 11 patients with single ventricle (SV) physiology who underwent staged surgical palliation.

\section{CPB and MUF}

CPB was established with ascending aortic and bicaval or single right atrial cannulation using a heart-lung machine (SIII roller pump, Stockert, Munich, Germany or MERA HAS heart-lung machine, Senko Medical Instruments, Tokyo, Japan) and an oxygenator (D901 or D902 Lilliput, Sorin Group Italia, Modena, Italy). The CPB flow was maintained at $150-180 \mathrm{ml} \cdot \mathrm{kg}^{-1} \cdot \mathrm{min}^{-1}$ for patients less than $10 \mathrm{~kg}$, and $2.4 \mathrm{~L} \cdot \mathrm{m}^{-2} \cdot \mathrm{min}^{-1}$ for patients over $10 \mathrm{~kg}$. Deep hypothermic circulatory arrest was used in 2 infants. Priming solution was prepared without blood for all patients except 2 small infants. Myocardial protection was obtained by intermittent antegrade crystalloid cardioplegia (Modified St Thomas' solution, $20 \mathrm{ml} / \mathrm{kg}$ at initial injection followed by $10 \mathrm{ml} / \mathrm{kg}$ for maintenance), which was given every $20 \mathrm{~min}$.

A polysulfon hemoconcentrator (Aquastream 04, JMS, Tokyo, Japan) was used. Arteriovenous MUF was performed immediately after termination of $\mathrm{CPB}$ with the technique described previously. ${ }^{2,16}$ A MUF circuit was established by connecting an accessory arterial line (Senko Medical Instruments or TERUMO Medical Corporation, Tokyo, Japan) to an aortic cannula as an inflow to the ultrafilter and a venous line was attached to a venous cannula as an outflow. Suction was applied to the filter port at a rate of no more than $100 \mathrm{mmHg}$. The endpoint was determined as either $15 \mathrm{~min}$ after the start of MUF, or the point at which the circuit contents were completely salvaged. The doses of catecholamine and vasodilator remained unchanged during MUF.

\section{Echocardiographic Evaluation}

Two-dimensional and Doppler color flow echocardiograms were obtained using a 5-MHz biplane esophageal probe transducer and an echocardiographic system (Sonos 5500 models; Philips Medical Systems, Andover, MA, USA or SSD 5500, Aloka, Tokyo, Japan). A miniature 3-8-MHz single-plane probe transducer (UST-5240S-5, Aloka) was used for patients weighting less than $3 \mathrm{~kg}$. The probe was inserted into the esophagus by an anesthesiologist after induction of general anesthesia, and remained throughout the procedure. Baseline measurement was performed after the chest opening in order to eliminate the effect of the chest wall and pericardium on cardiac function. The pre-MUF measurement was performed during the period of approximately 2 min between termination of CPB and initiation of MUF and the post-MUF measurement was obtained shortly after termination of MUF and completed before chest closure. Hemodynamics including blood pressure, central venous pressure (CVP), and heart rate, and hematocrit were recorded at the same time.

The MPI is defined as (a-b)/b, where ' $a$ ' is the interval between the end and onset of systemic ventricular inflow, and ' $b$ ' is the ejection time of a systemic outflow. ${ }^{9}$ The normal value of MPI that we used is $0.30 \pm 0.08$ in the LV and $0.26 \pm 0.08$ in the right ventricle. ${ }^{11}$ The $\%$ change in MPI between the pre- and post-CPB and between the pre-and post-MUF values was calculated as $\left\{\left[\left(\mathrm{MPI}_{\text {pre-CPB }}\right.\right.\right.$ (MUF) $)-$

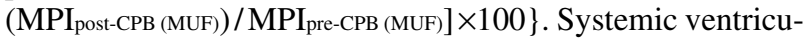
lar end-diastolic (SVEDd) and end-systolic diameters (SVESd), and systemic ventricular posterior wall thickness in diastole were obtained in the short-axis view, and fractional shortening (FS) was calculated as $\{[($ SVEDd-SVESd $)$ / SVEDd] $\times 100\}$. The peak velocity of passive mitral filling (E), peak velocity of active mitral filling (A), the ratio of passive to active velocity (E/A), and deceleration time were 


\begin{tabular}{|c|c|c|c|c|c|}
\hline & Group & Pre-CPB & Pre-MUF & Post-MUF & $P$ value \\
\hline \multirow[t]{5}{*}{ SBP $(\mathrm{mmHg})$} & ASD & $95 \pm 14$ & $77 \pm 8$ & $99 \pm 8$ & $0.0071 *, \uparrow$ \\
\hline & VSD & $91 \pm 12$ & $69 \pm 10$ & $94 \pm 16$ & $0.001^{*, \uparrow}$ \\
\hline & TOF & $87 \pm 15$ & $64 \pm 7$ & $88 \pm 9$ & $0.0005^{*}, \uparrow$ \\
\hline & SLV & $86 \pm 9$ & $68 \pm 12$ & $88 \pm 13$ & $0.005^{\star}, \pi$ \\
\hline & SRV & $80 \pm 15$ & $64 \pm 11$ & $84 \pm 12$ & $0.005^{\star}, \pi$ \\
\hline \multirow[t]{5}{*}{$\mathrm{CVP}(\mathrm{mmHg})$} & ASD & $6.1 \pm 2.8$ & $8.1 \pm 2.0$ & $6.1 \pm 2.4$ & $0.0053^{\natural}$ \\
\hline & VSD & $6.8 \pm 1.2$ & $9.2 \pm 1.3$ & $6.5 \pm 2.0$ & $0.0083^{*}, \pi$ \\
\hline & TOF & $5.7 \pm 2.9$ & $9.4 \pm 1.8$ & $8.2 \pm 2.7$ & $0.0018^{*, \pi}$ \\
\hline & SLV & $10.8 \pm 3.3$ & $10 \pm 4.1$ & $9.2 \pm 4.5$ & NS \\
\hline & SRV & $11.8 \pm 4$ & $12 \pm 5.1$ & $11 \pm 4.4$ & NS \\
\hline \multirow[t]{5}{*}{ HR (beats/min) } & ASD & $104 \pm 24$ & $116 \pm 14$ & $110 \pm 13$ & NS \\
\hline & VSD & $111 \pm 19$ & $122 \pm 30$ & $115 \pm 13$ & NS \\
\hline & TOF & $114 \pm 20$ & $133 \pm 18$ & $134 \pm 16$ & NS \\
\hline & SLV & $101 \pm 18$ & $127 \pm 7$ & $122 \pm 8$ & $0.0384^{\star}$ \\
\hline & SRV & $118 \pm 18$ & $127 \pm 24$ & $124 \pm 26$ & $0.0264^{\star}$ \\
\hline \multirow[t]{5}{*}{ Hematocrit (\%) } & ASD & $35 \pm 2$ & $22 \pm 3$ & $32 \pm 3$ & $<0.0001^{*}, 9$ \\
\hline & VSD & $37 \pm 3$ & $20 \pm 4$ & $34 \pm 6$ & $<0.0001^{*}$, \\
\hline & TOF & $41 \pm 3$ & $21 \pm 2$ & $35 \pm 4$ & $<0.0001^{*}$, १ \\
\hline & SLV & $40 \pm 5$ & $22 \pm 3$ & $34 \pm 3$ & $<0.0001^{*}$, \\
\hline & SRV & $42 \pm 7$ & $23 \pm 4$ & $34 \pm 5$ & $<0.0001^{*, \pi}$ \\
\hline
\end{tabular}

${ }^{*} \mathrm{P}<0.05$ Pre-CPB vs Pre-MUF; १P $<0.05$ Pre-MUF vs Post-MUF.

$\mathrm{SBP}$, systolic blood pressure; CVP, central venous pressure; HR, heart rate. Other abbreviations see in Table 1.

\begin{tabular}{|lccll|}
\hline Table 3. & Myocardial Performance Index Before and After MUF & & \\
Group & Pre-CPB & Pre-MUF & Post-MUF & P value \\
ASD & $0.421 \pm 0.25$ & $0.475 \pm 0.21$ & $0.242 \pm 0.06$ & $0.04 \pi$ \\
VSD & $0.283 \pm 0.09$ & $0.398 \pm 0.16$ & $0.276 \pm 0.07$ & $0.05^{*, \pi}$ \\
TOF & $0.298 \pm 0.16$ & $0.370 \pm 0.12$ & $0.225 \pm 0.05$ & $0.04^{*, \pi}$ \\
SLV & $0.288 \pm 0.12$ & $0.326 \pm 0.08$ & $0.257 \pm 0.06$ & $0.004^{\pi}$ \\
SRV & $0.346 \pm 0.13$ & $0.402 \pm 0.11$ & $0.310 \pm 0.09$ & $0.02^{\Uparrow}$ \\
\hline
\end{tabular}

${ }^{*} \mathrm{P}<0.05$ Pre-CPB vs Pre-MUF; १P $<0.05$ Pre-MUF vs Post-MUF.

Abbreviations see in Table 1.

measured in the angulated 4-chamber view.

\section{Inter- and Intraobserver Variability}

Two investigators independently reviewed 15 videotapes on 2 occasions each. Each investigator was unaware of the patient's profiles. Videotapes were masked and displayed in a random order. There was reasonable interobserver $(\mathrm{r}=0.933$, $\mathrm{P}<0.01)$ and intraobserver agreement (Observer 1: $\mathrm{r}=0.958$, $\mathrm{P}<0.001$; Observer 2: $\mathrm{r}=0.943, \mathrm{P}<0.001)$ in calculating the MPI.

\section{Statistical Analysis}

Differences between groups were determined by 1-way analysis of variance (ANOVA) followed by Bonferroni multiple comparisons test. The level of statistical significance was set at $\mathrm{P}=0.05$. Data are presented as the mean \pm standard deviation and NS indicates statistical insignificance. Correlations between MPI and aortic cross-clamping or CPB time and the inter- and intra-observer relationships were analyzed using linear regression by Spearman's correlation test.

\section{Results}

There were no deaths or MUF-related complications in the study group. The aortic cross-clamping (AoX) and CPB times of the TOF group were longer than those of the other groups (Table 1). Four patients were transfused blood either in priming $(n=2)$ or during the procedure $(n=2)$. All patients were in sinus rhythm at the time of the ITEE study.

Hemodynamic results are shown in Table 2. Systolic blood pressure decreased after CPB and increased after MUF in all groups $(\mathrm{P}<0.01$ for all groups). CVP increased after $\mathrm{CPB}$ and significantly decreased after MUF in the 3 groups that had 2 -ventricle physiology $(\mathrm{P}<0.01)$. There was minimal change in the CVP of the single ventricle groups (Table 2).

\section{Global Ventricular Performance}

The MPI increased at termination of CPB in all groups, which was statistically significant in the VSD and TOF groups (Table 3). The \% increase in MPI after CPB was higher in the TOF group than in the SLV group (Figure 1A). MUF resulted in a significant improvement in MPI in all groups $(\mathrm{P}<0.05$ for all groups, Table 3$)$. The \% improvement in MPI after MUF was also larger in the TOF group than in the other groups, but was not statistically significant (Figure 1B). There was no correlation between either the AoX or the CPB time and the degree of impairment in MPI after CPB (Figures 2A,B). Conversely, there was a weak negative 

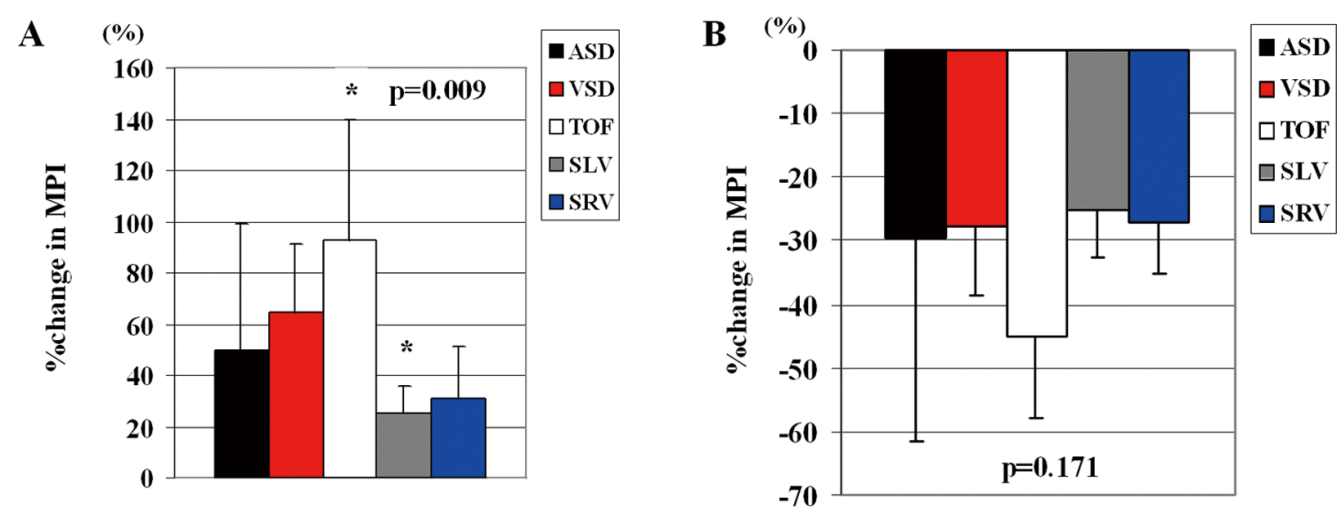

Figure 1. (A) The \% change in myocardial performance index (MPI) of the systemic ventricles after cardiopulmonary bypass (CPB) compared with baseline; ${ }^{*}<0.05$. (B) The \% change in MPI of the systemic ventricles after modified ultrafiltration (MUF) compared with pre-MUF values. ASD, atrial septal defect; VSD, ventricular septal defect; TOF, tetralogy of Fallot; SLV, single left ventricle; SRV, single right ventricle.
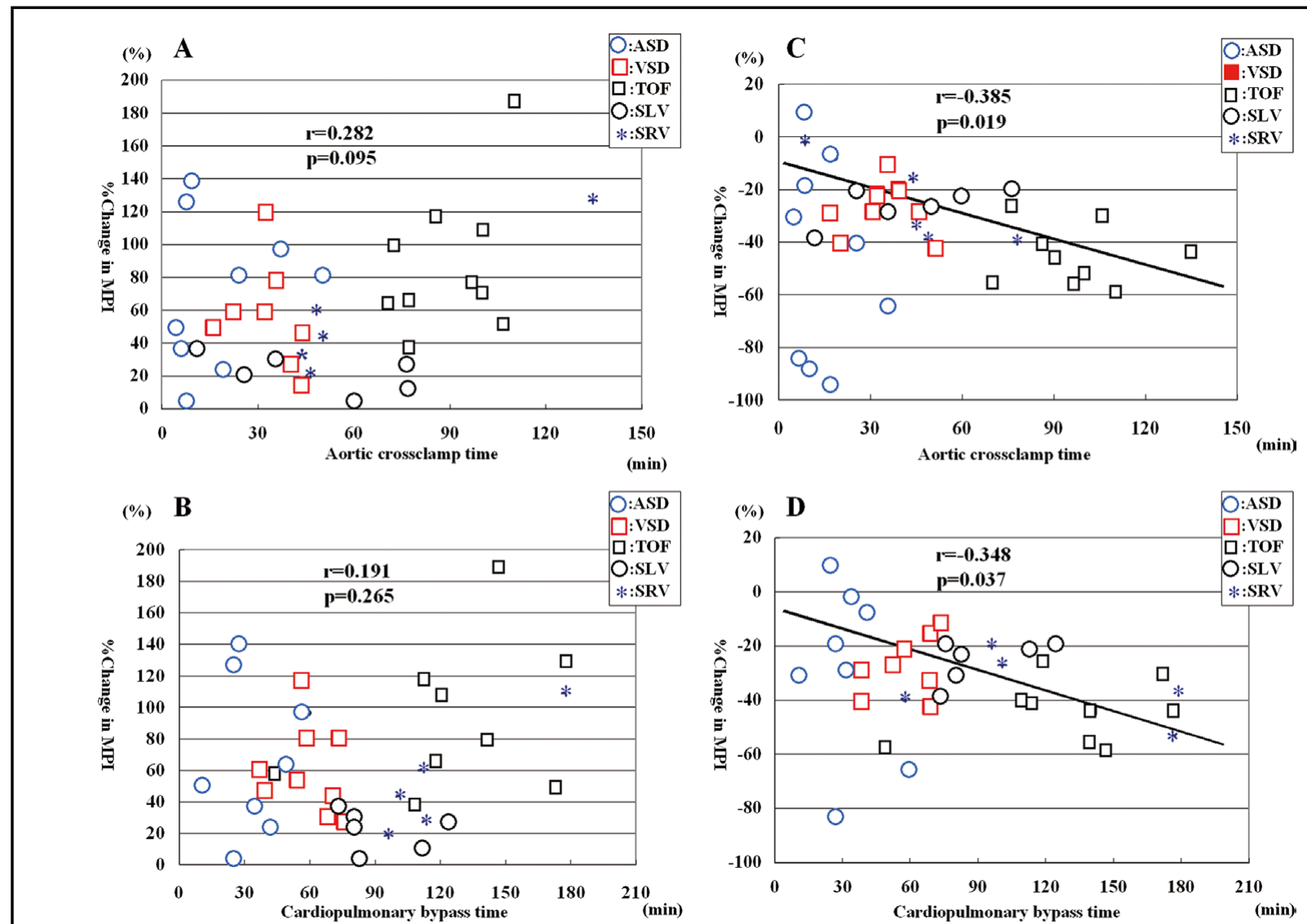

Figure 2. (A, B) Relationship between the aortic cross-clamping (AoX) time and the \% increase in MPI after CPB. (C,D) Relationship between CPB time and the \% decrease MPI after MUF. Other abbreviations see in Figure 1.

correlation between the duration of AoX and $\mathrm{CPB}$, and the degree of improvement in MPI $(\mathrm{r}=-0.385, \mathrm{P}=0.019 ; \mathrm{r}=-0.348$, $\mathrm{P}=0.037$, respectively) (Figures 2C, D).

Systolic and diastolic functions before and after MUF are summarized in Tables 4 and 5. MUF improved ventricular systolic function, including FS and ejection time $(\mathrm{P}<0.05$ for all groups). There was minimal change in ventricular posterior wall thickness after MUF in all patients $(\mathrm{P}=\mathrm{NS})$. There were significant impairments in diastolic function in the VSD and TOF groups, and these were improved by MUF. 


\begin{tabular}{|c|c|c|c|c|c|}
\hline & Group & Pre-CPB & Pre-MUF & Post-MUF & $P$ value \\
\hline \multirow[t]{5}{*}{ FS (\%) } & ASD & $32 \pm 4$ & $34 \pm 4$ & $40 \pm 7$ & $0.037 \pi$ \\
\hline & VSD & $28 \pm 3$ & $32 \pm 1$ & $38 \pm 7$ & $0.016 \pi$ \\
\hline & TOF & $32 \pm 7$ & $30 \pm 3$ & $34 \pm 4$ & $0.004 \pi$ \\
\hline & SLV & $32 \pm 3.4$ & $28 \pm 5$ & $33 \pm 7$ & $0.022^{*, n}$ \\
\hline & SRV & $28 \pm 6$ & $26 \pm 5$ & $30 \pm 6$ & $0.044 \pi$ \\
\hline \multirow[t]{5}{*}{ Ejection time (ms) } & ASD & $233 \pm 24$ & $216 \pm 33$ & $239 \pm 18$ & $0.019^{*}$ \\
\hline & VSD & $261 \pm 28$ & $199 \pm 17$ & $242 \pm 21$ & $0.002^{*, 9}$ \\
\hline & TOF & $218 \pm 18$ & $188 \pm 27$ & $209 \pm 22$ & $0.185^{\star}$ \\
\hline & SLV & $252 \pm 47$ & $189 \pm 39$ & $225 \pm 26$ & $0.005^{\star, n}$ \\
\hline & SRV & $240 \pm 23$ & $198 \pm 29$ & $203 \pm 16$ & $0.018^{*}$ \\
\hline \multirow[t]{5}{*}{ SVPWTd (mm) } & ASD & $5.7 \pm 1.2$ & $6.5 \pm 1.7$ & $6.6 \pm 1.7$ & NS \\
\hline & VSD & $5.8 \pm 0.8$ & $6.4 \pm 0.9$ & $6.1 \pm 1.2$ & NS \\
\hline & TOF & $4.1 \pm 0.4$ & $4.6 \pm 0.2$ & $4.3 \pm 0.2$ & $0.0197^{*}$ \\
\hline & SLV & $4.4 \pm 0.2$ & $5.1 \pm 0.2$ & $5.1 \pm 0.3$ & $0.0135^{*}$ \\
\hline & SRV & $4.9 \pm 0.5$ & $5.2 \pm 0.4$ & $5.2 \pm 0.5$ & NS \\
\hline
\end{tabular}

${ }^{*} \mathrm{P}<0.05$ Pre-CPB vs Pre-MUF; १P $<0.05$ Pre-MUF vs Post-MUF.

FS, fractional shortening; SVPWTd, systemic ventricular posterior wall thickness in diastole. Other abbreviations see in Table 1.

\begin{tabular}{|c|c|c|c|c|c|}
\hline & Group & Pre-CPB & Pre-MUF & Post-MUF & $P$ value \\
\hline \multirow[t]{5}{*}{$E(\mathrm{~cm} / \mathrm{s})$} & ASD & $93 \pm 16$ & $96 \pm 31$ & $91 \pm 11$ & NS \\
\hline & VSD & $104 \pm 22$ & $81 \pm 27$ & $88 \pm 23$ & $<0.0001^{*}$, n \\
\hline & TOF & $89 \pm 37$ & $80 \pm 25$ & $96 \pm 30$ & NS \\
\hline & SLV & $76 \pm 20$ & $82 \pm 34$ & $98 \pm 33$ & NS \\
\hline & SRV & $80 \pm 32$ & $81 \pm 12$ & $93 \pm 12$ & $0.0351 \pi$ \\
\hline \multirow[t]{5}{*}{$\mathrm{A}(\mathrm{cm} / \mathrm{s})$} & ASD & $77 \pm 18$ & $102 \pm 23$ & $86 \pm 7$ & NS \\
\hline & VSD & $65 \pm 15$ & $75 \pm 20$ & $65 \pm 17$ & NS \\
\hline & TOF & $69 \pm 15$ & $87 \pm 28$ & $79 \pm 35$ & NS \\
\hline & SLV & $55 \pm 17$ & $72 \pm 8$ & $65 \pm 16$ & $0.0512^{*}$ \\
\hline & SRV & $63 \pm 17$ & $88 \pm 18$ & $80 \pm 8$ & $0.0554^{\star}$ \\
\hline \multirow[t]{5}{*}{$E / A$} & ASD & $1.24 \pm 0.24$ & $0.95 \pm 0.17$ & $1.05 \pm 0.11$ & $0.0043^{*}$ \\
\hline & VSD & $1.63 \pm 0.40$ & $1.14 \pm 0.08$ & $1.48 \pm 0.27$ & $0.0159^{*}, 9$ \\
\hline & TOF & $1.28 \pm 0.45$ & $0.95 \pm 0.21$ & $1.20 \pm 0.14$ & $0.0124^{*}, 9$ \\
\hline & SLV & $1.55 \pm 0.58$ & $1.12 \pm 0.41$ & $1.51 \pm 0.45$ & NS \\
\hline & SRV & $1.12 \pm 0.31$ & $1.07 \pm 0.35$ & $1.15 \pm 18$ & NS \\
\hline \multirow[t]{5}{*}{ Deceleration time (ms) } & ASD & $100 \pm 49$ & $93 \pm 11$ & $96 \pm 10$ & NS \\
\hline & VSD & $92 \pm 24$ & $107 \pm 28$ & $89 \pm 33$ & $0.0011^{*, 9}$ \\
\hline & TOF & $85 \pm 13$ & $71 \pm 12$ & $52 \pm 11$ & $0.0488^{\Uparrow}$ \\
\hline & SLV & $89 \pm 8$ & $101 \pm 9$ & $97 \pm 7$ & NS \\
\hline & SRV & $97 \pm 22$ & $112 \pm 16$ & $95 \pm 10$ & NS \\
\hline
\end{tabular}

${ }^{*} \mathrm{P}<0.05$ Pre-CPB vs Pre-MUF; $1 \mathrm{P}<0.05$ Pre-MUF vs Post-MUF.

$E(A)$, peak velocity of the early (atrial) filling; $E / A$, ratio of $E$ to $A$. Other abbreviations see in Table 1.

\section{Discussion}

Using MPI as a parameter of global ventricular performance, we analyzed the diagnosis-based differences in the response of the systemic ventricles to MUF in 5 different groups of children undergoing open-heart surgery. As opposed to previous studies in which the MUF group was compared with controls, ${ }^{3,6,7}$ our study compared the pre-MUF value to the post-MUF value in each patient, thereby allowing us to document how MUF improves cardiac function in an individual heart. The results are as follows: (1) MUF significantly decreased MPI in all subgroups, indicating that MUF improved systemic ventricular function; (2) negative correlation between AoX and CPB times, and the \% change in MPI indicated that MUF is more effective when a patient is exposed to longer myocardial ischemia and CPB; and (3) MUF attenuated abnormal relaxation in the VSD and TOF groups.

Ventricular Response to MUF: Diagnosis-Based Difference There were considerable differences among the 5 groups regarding the response of the systemic ventricles to MUF. The ASD group had minimal global, as well as systolic and diastolic, dysfunction after CPB because of the short AoX 
and CPB times; therefore, we found that MUF had only a minimal effect on cardiac function in this group. Conversely, we found greater improvement in global ventricular function, as well as systolic and diastolic function, in the VSD and TOF groups, which had relatively longer AoX times and were known to have myocardial dysfunction after CPB. ${ }^{17}$ Furthermore, this study found a negative correlation between the duration of AoX and CPB and the \%change in MPI, indicating that MUF improves global cardiac function more effectively in patients undergoing lengthy surgery than in patients undergoing shorter surgery. We hypothesize that the diagnosis-based differences in the recovery rates of MPI in response to MUF seem not to be directly related to pre- or postoperative cardiac morphology or physiology (ie, the degree of volume overload to the systemic ventricles after surgical correction), but rather are simply related to the length of exposure to extracorporeal circulation and subsequent myocardial and systemic edema. In this sense, there is minimal benefit of MUF on ventricular function in patients undergoing relatively simple surgery.

\section{MPI}

The MPI has many advantages in assessing the effect of MUF in children with congenital heart diseases. The index is (1) less dependent on HR and blood pressure; 9 (2) independent of the geometry of the ventricles, so it can be used to assess any type of ventricle; $;^{10-13}$ and (3) an efficient quantitative assessment of the ventricle, which is ideal for comparing the relative ventricular performance before and after various types of interventions. ${ }^{14,15}$ A striking disadvantage, however, is that the MPI may be affected by loading conditions. ${ }^{18} \mathrm{CVP}$ changes dramatically during the period from termination of CPB to MUF, and in fact, was higher at termination of CPB and dropped significantly after MUF in the biventricular repair groups. Because we did not measure LA pressure, we never knew the real LV preload. Therefore, systemic ventricular preload during MUF and its subsequent effects on MPI are unknown. Although improvement in the MPI after MUF was fairly consistent with improvement in hemodynamics and systolic function, it is possible the changes in the various echocardiographic parameters seen in this study may have been partly affected by the loading conditions.

\section{Mechanism of MUF-Induced Improvement in Ventricular Function}

Reduction of myocardial edema is one of the most important mechanisms accounting for the improvement in cardiac function by MUF. ${ }^{3}$ Daggett et al showed a significantly lower ventricular weight in the MUF group than the control group of piglets, indicating that MUF lessened myocardial edema. ${ }^{19}$ Gaynor et al also showed reduced myocardial edema in a MUF group as evaluated by echocardiography. ${ }^{20}$ No significant improvement in myocardial edema was noted in our study. Removal of potential myocardial depressants, including cytokines, is another proposed mechanism by which MUF may 'prevent' myocardial dysfunction in the early postoperative period. ${ }^{3,19}$ Such a mechanism, however, might play only a minor role in actively 'improving' cardiac function, as seen in the present study. Rather, we hypothesize that MUF-induced hemoconcentration is a more important mechanism of improving ventricular performance in such a short period of time. Martine et al revealed that in a hamster model mild hemoconcentration and increased viscosity of blood augmented cardiac output and microvascular blood flow in the myocardium, and improved oxygen delivery. ${ }^{21}$ Other potential mechanisms include optimization of preload and improved oxygen delivery to peripheral tissues, as shown by increased hematocrit, as well as reduction of systemic edema, which might favorably alter systemic vascular resistance and afterload.

\section{Effect of MUF on Diastolic Function}

Contradictory results have been reported regarding the effect of MUF on diastolic function. Davies et al showed improved ventricular compliance in the MUF group, ${ }^{7}$ whereas Charturvedi et al observed no improvement in diastolic function after MUF, and suggested that MUF may not have removed a sufficient amount of the excess myocardial tissue water to improve diastolic function. ${ }^{8}$ Chaturvedi et al used a conductance catheter to assess left ventricular dysfunction after repair of simple congenital heart defects such as ASD, and found minimal diastolic dysfunction, despite the fact that systolic dysfunction was found after even a short period of AoX. ${ }^{22}$ This phenomenon may explain the controversial results of the effect of MUF on diastolic function. The present study showed only a minimal advantage of MUF on diastolic function in the ASD group, whereas an improvement in abnormal relaxation was found in the TOF and VSD groups, indicating that MUF has the potential to improve diastolic function by removing excess fluid if the myocardium is considerably edematous as a result of long Aox and CPB times.

\section{Study Limitations}

The important limitation of this study is its design. Because we compared the pre-MUF and post-MUF values in individual patients, and there was no control group without MUF treatment, we cannot confirm whether the functional recovery seen in this study was induced by MUF or by the spontaneous recovery of ventricular function that occurs early after CPB. ${ }^{23}$

\section{Conclusions}

MUF ameliorated the MPI in pediatric patients with all types of congenital heart defects, indicating improved global systemic ventricular function, although the effect of altered preload on changes in MPI is uncertain. The recovery rate of MPI differed among the patient groups. MUF may be particularly useful for restoring global ventricular performance in high-risk patients being exposed to longer extracorporeal circulation, but not for patients undergoing relatively simple and short surgery.

\section{References}

1. Kirklin JK, Blackstone EH, Kirklin JW. Cardiopulmonary bypass: Studies on its damaging effects. Blood Purif 1987; 5: 168-178.

2. Naik SK, Knight A, Elliott MJ. A successful modification of ultrafiltration for cardiopulmonary bypass in children. Perfusion 1991; 6: $41-50$.

3. Gaynor JW. The effect of modified ultrafiltration on the postoperative course in patients with congenital heart disease. Semin Thorac Cardiovasc Surg Pediatr Card Surg Annu 2003; 6: 128-139.

4. Berdat PA, Eichenberger E, Ebell J, Pfammatter JP, Pavlovic M, Zobrist $\mathrm{C}$, et al. Elimination of proinflammatory cytokines in pediatric cardiac surgery: Analysis of ultrafiltration method and filter type. J Thorac Cardiovasc Surg 2004; 127: 1688-1696.

5. Mahmoud ABS, Burhami MS, Hannef AA, Jamjoom AA, AlGithmi IS, Baslaim GM. Effect of modified ultrafiltration on pulmonary function after cardiovascular bypass. Chest 2005; 128: $3447-3453$.

6. Naik SK, Knight A, Elliott M. A prospective randomized study of a modified technique of ultrafiltration during pediatric open-heart 
surgery. Circulation 1991; 84(Suppl 6): III-422-III-434.

7. Davies MJ, Nguyen K, Gaynor JW, Elliott MJ. Modified ultrafiltration improves left ventricular systolic function in infants after cardiopulmonary bypass. J Thorac Cardiovasc Surg 1998; 115: $361-369$.

8. Chaturvedi RR, Shore DF, White PA, Scallan MH, Gothard JW, Redington AN, et al. Modified ultrafiltration improves global left ventricular systolic function after open-heart surgery in infants and children. Eur J Cardiothorac Surg 1999; 15: 742-746.

9. Tei C. New noninvasive index for combining systolic and diastolic function. J Cardiol 1995; 26: 135-136.

10. Tei C, Dujardin KS, Hodge DO, Bailey KR, McGoon MD, Tajik AJ, et al. Doppler echocardiographic index for assessment of global right ventricular function. J Am Soc Echocardiogr 1996; 9: $838-847$.

11. Zhang YQ, Sun K, Zhu SL, Wu LP, Chen GZ, Zhang ZF, et al. Doppler myocardial performance index in assessment of ventricular function in children with single ventricle. World J Pediatr 2008; 4: $109-113$.

12. Salehian O, Schwerzmann M, Merchant N, Webb GD, Siu SC, Therrien J. Assessment of systemic right ventricular function in patients with transposition of the great arteries using the myocardial performance index: Comparison with cardiac magnetic resonance imaging. Circulation 2004; 110: 3229-3233.

13. Williams RV, Ritter S, Tani LY, Pagoto LT, Minich LL. Quantitative assessment of ventricular function in children with single ventricles using the Doppler myocardial performance index. Am J Cardiol 2000; 86: 1106-1110.

14. Bein B, Renner J, Caliebe D, Scholz J, Paris A, Fraund S, et al. Sevoflurane but not propofol preserves myocardial function during minimally invasive direct coronary artery bypass surgery. Anesth Analg 2005; 100: 610-616.

15. Murphy GS, Marymont JH, Szokol JW, Avram MJ, Vender JS. Correlation of the myocardial performance index with conventional echocardiographic indices of systolic and diastolic function: A study in cardiac surgical patients. Echocardiography 2007; 24:
$26-33$.

16. Kotani Y, Honjo O, Osaki S, Kawabata T, Ugaki S, Fujii Y, et al. Effect of modified ultrafiltration on postoperative course in neonates with complete transposition of the great arteries undergoing arterial switch operation. Circ J 2008; 72: 1476-1480.

17. Cheung MM, Smallhorn JF, Vogel M, Van Arsdell G, Redington AN. Disruption of the ventricular myocardial force-frequency relationship after cardiac surgery in children: Noninvasive assessment by means of tissue Doppler imaging. $J$ Thorac Cardiovasc Surg 2006; 131: 625-631.

18. Moller JE, Poulsen SH, Egstrup K. Effect of preload alternations on a new Doppler echocardiographic index of combined systolic and diastolic performance. J Am Soc Echocardiogr 1999; 12: $1065-1072$.

19. Daggett CW, Lodge AJ, Scarborough JE, Chai PJ, Jaggers J, Ungerleider RM. Modified ultrafiltration versus conventional ultrafiltration: A randomized prospective study in neonatal piglets. $J$ Thorac Cardiovasc Surg 1998; 115: 336-341.

20. Gaynor JW, Tulloh RMR, Owen CH, Sullivan ID, Elliott MJ. Modified ultrafiltration reduces myocardial edema and reverses hemodilution following cardiopulmonary bypass in children. $J \mathrm{Am}$ Coll Cardiol 1995; 25: 200A.

21. Martini J, Tsai AG, Cabrales P, Johnson PC, Intaglietta M. Increased cardiac output and microvascular blood flow during mild hemoconcentration in hamster window model. Am J Physiol Heart Circ Physiol 2006; 291: H310-H317.

22. Chaturvedi RR, Lincoln C, Gothard JW, Scallan MH, White PA, Redington AN, et al. Left ventricular dysfunction after open repair of simple congenital heart defects in infants and children: Quantitation with the use of a conductance catheter immediately after bypass. J Thorac Cardiovasc Surg 1998; 115: 77-83.

23. De Hert SG, Rodrigus IE, Haenen LR, De Mulder PA, Gillebert TC. Recovery of systolic and diastolic left ventricular function early after cardiopulmonary bypass. Anesthesiology 1996; 85: $1063-1075$. 